$$
\begin{array}{lllll}
\lrcorner & \lrcorner
\end{array}
$$

\title{
The Chow ring of double EPW sextics
}

\lrcorner$\quad$ Andrea Ferretti

\lrcorner

\rfloor

. 


\title{
The Chow ring of double EPW sextics
}

\author{
Andrea Ferretti
}

A conjecture of Beauville and Voisin states that for an irreducible symplectic variety $X$ the subring of $C H^{*}(X)$ generated by divisors goes injectively into the cohomology of $X$, via the cycle map. We prove this for a very general double Eisenbud-Popescu-Walter sextic.

\section{Introduction}

A difficult problem in algebraic geometry is to characterize the kernel (and the image) of the cycle map

$$
c: C H^{*}(X) \rightarrow H^{*}(X, \mathbb{Z})
$$

for a smooth projective variety $X$ over $\mathbb{C}$. When $X$ is an irreducible symplectic variety there is this general conjecture:

Conjecture 1 [Beauville 2007]. Let $X$ be an irreducible symplectic variety, and let $\mathrm{DCH}(X) \subset C H_{\mathbb{Q}}^{*}(X)$ be the subalgebra generated by the divisors. Then the cycle map

$$
c: C H^{*}(X)_{\mathbb{Q}} \rightarrow H^{*}(X, \mathbb{Q})
$$

is injective when restricted to $D C H(X)$.

We refer to the original article for the motivation of the conjecture and its link with the conjectures of Bloch and Beilinson; we just remark that the conjecture was known to hold when $X$ is a K3 surface from [Beauville and Voisin 2004]. Conjecture 1 explicitly means the following: any polynomial relation

$$
P\left(D_{1}, \ldots, D_{k}\right)=0
$$

in the fundamental classes of divisors which holds in $H^{*}(X)$ already holds inside $C H_{\mathbb{Q}}^{*}(X)$.

This has been extended by Claire Voisin:

I'd like to thank K. O'Grady for his constant support, advice, and encouragement during my Ph.D.. MSC2010: primary 53C26; secondary 14C15, 14J35.

Keywords: EPW sextics, Chow ring, hyperkähler, irreducible symplectic varieties. 
Conjecture 2 [Voisin 2008]. Let X be an irreducible symplectic variety. Any polynomial relation

$$
P\left(D_{1}, \ldots, D_{k}, c_{i}(X)\right)=0
$$

in the fundamental classes of divisors and in the Chern classes of $X$ that holds in $H^{*}(X)$ already holds in $\mathrm{CH}_{\mathbb{Q}}^{*}(X)$.

Theorem [Voisin 2008]. Conjecture 2 holds when

- $X=S^{[n]}$, for some K3 surface $S$, and $n \leq 2 b_{2}(S)_{\mathrm{tr}}+4$, where $b_{2}(S)_{\mathrm{tr}}$ is the rank of the transcendental part of $H^{2}(S)$, that is, the orthogonal of the Néron-Severi lattice, or

- $X$ is the Fano variety of lines on a cubic fourfold $Y \subset \mathbb{P}^{5}$.

To our knowledge, no other cases of the conjecture have been verified. We prove it to be true for double EPW sextics, a class of irreducible symplectic varieties introduced in [O'Grady 2008a] and named after Eisenbud, Popescu and Walter. (We will review their construction shortly.) More precisely:

Theorem 1.1. Let $X$ be a double EPW sextic and $f: X \rightarrow Y$ its associated double covering. Let

$$
h=f^{*} \mathscr{O}_{Y}(1)
$$

be the natural polarization. Then every polynomial relation between $h$ and the Chern classes of $X$ which holds in $H^{*}(X, \mathbb{Q})$ already holds in $C^{*}(X)_{\mathbb{Q}}$.

In particular, if $X$ is very general, Conjecture 2 holds for $X$.

Theorem 1.1 is the main result of my Ph.D. thesis [Ferretti 2009]. Some facts that are only cited in the present paper are described there in more detail.

EPW sextics. In this section we recall some known facts about EPW sextics and we fix the notation that we shall use. The results here are from [O'Grady 2006; 2008a]; see also [Ferretti 2009] for a detailed introduction.

We start with a six-dimensional vector space $V$ over the field $\mathbb{C}$. The space $\wedge^{6} V$ is one-dimensional, so we choose once and for all an isomorphism

$$
\text { vol : } \bigwedge^{6} V \rightarrow \mathbb{C}
$$

This endows $\wedge^{3} V$ with a symplectic form, given by

$$
(\alpha, \beta)=\operatorname{vol}(\alpha \wedge \beta)
$$

for $\alpha, \beta \in \bigwedge^{3} V$, so $\bigwedge^{3} V$ becomes a symplectic vector space of dimension 20 .

For each nonzero $v \in V$ we can consider the Lagrangian subspace

$$
F_{v}=\left\{v \wedge \alpha \mid \alpha \in \bigwedge^{2} V\right\} .
$$


This is clearly isotropic, and the isomorphism

$$
\begin{aligned}
\varphi_{v} & : F_{v} \stackrel{\cong}{\longmapsto} \wedge^{2}(V /\langle v\rangle) \\
v & \wedge \alpha \longmapsto[\alpha]
\end{aligned}
$$

shows that $\operatorname{dim} F_{v}=\left(\begin{array}{l}5 \\ 2\end{array}\right)=10$.

Since the subspace $F_{v}$ only depends on the class $[v] \in \mathbb{P}(V)$, the subspaces $F_{v}$ fit together, giving rise to a Lagrangian subbundle $F$ of the trivial symplectic bundle $\mathbb{P}(V) \times \wedge^{3} V$. The maps in (1-1) then yield an isomorphism

$$
F \cong \mathscr{Y} \otimes \bigwedge^{2} \mathscr{Q}
$$

where 2 is the tautological quotient bundle on $\mathbb{P}(V)$ and $\mathscr{Y}$ the tautological subbundle. From this a standard computation gives

$$
c_{1}(F)=c_{1}\left(\bigwedge^{2} 2\right)+\operatorname{rk}(F) c_{1}(\mathscr{Y})=-6 H,
$$

where $H=c_{1}(\mathcal{O}(1))$ is the hyperplane class on $\mathbb{P}(V)$.

We are now ready to define the EPW sextics, as follows. Fix a Lagrangian subspace $A \subset \wedge^{3} V$. Note that the symplectic form gives a canonical identification

$$
\wedge^{3} V / A \cong A^{\vee} \text {. }
$$

Let

$$
\lambda_{A}: F \rightarrow \mathcal{O}_{\mathbb{P}(V)} \otimes A^{\vee}
$$

be the inclusion $F \hookrightarrow \mathbb{O}_{\mathbb{P}(V)} \otimes \bigwedge^{3} V$ followed by the projection modulo $A$. The map $\lambda_{A}$ is a map of vector bundles of equal rank 10 .

Definition 1.2. We set

$$
Y_{A}=Z\left(\operatorname{det} \lambda_{A}\right)
$$

the zero locus of the determinant of $\lambda_{A}$. This is a subscheme of $\mathbb{P}(V)$; when it is not the whole of $\mathbb{P}(V), Y_{A}$ is called an EPW sextic. (It is indeed a sextic by (1-2).)

The support of the scheme $Y_{A}$ is by definition the locus

$$
\left\{[v] \in \mathbb{P}(V) \mid \operatorname{dim}\left(F_{v} \cap A\right) \geq 1\right\} .
$$

We then set

$$
Y_{A}[k]=\left\{[v] \in \mathbb{P}(V) \mid \operatorname{dim}\left(F_{v} \cap A\right) \geq k\right\},
$$

so that $Y_{A}=Y_{A}[1]$, at least set-theoretically. The loci $Y_{A}[k]$ also have a natural scheme structure, given by the vanishing of the determinants of the $(11-k) \times$ $(11-k)$ minors of $\lambda_{A}$.

The natural parameter space for EPW sextics is the Lagrangian Grassmannian $\mathbb{L} \mathbb{G}\left(\bigwedge^{3} V\right)$, or more precisely the Zariski open set parametrizing those $A$ for which 
$Y_{A} \subsetneq \mathbb{P}(V)$. We recall that $\mathbb{L} \mathbb{G}\left(\bigwedge^{3} V\right) \subset \operatorname{Gr}\left(10, \bigwedge^{3} V\right)$ is the subvariety of Lagrangian subspaces; it is a smooth variety of dimension 55 .

Double EPW sextics. Assume that $Y_{A}$ is not the whole of $\mathbb{P}(V)$. The map of vector bundles $\lambda_{A}$ in (1-3) is an injective homomorphism of sheaves, whose cokernel is supported on $Y_{A}$. If we denote by

$$
i_{A}: Y_{A} \rightarrow \mathbb{P}(V)
$$

the inclusion, then we have an exact sequence

$$
0 \longrightarrow F \longrightarrow O_{\mathbb{P}(V)} \otimes A^{\vee} \longrightarrow i_{A *}\left(\xi_{A}\right) \longrightarrow 0
$$

for some sheaf $\xi_{A}$ on $Y_{A}$. For a generic Lagrangian subspace $A$ the locus

$$
Y_{A}[2]=\left\{[v] \in \mathbb{P}(V) \mid \operatorname{dim}\left(F_{v} \cap A\right) \geq 2\right\}
$$

is properly contained in $Y_{A}$; it follows that $\xi_{A}$ is generically free of rank 1 . If

$$
\zeta_{A}=\xi_{A}^{\vee}(3),
$$

there is a natural multiplication map

$$
m_{A}: \zeta_{A} \otimes \zeta_{A} \rightarrow \mathrm{O}_{Y_{A}}
$$

More precisely:

Lemma 1.3 [O'Grady 2008a]. The map $m_{A}$ is symmetric and associative, and gives an isomorphism between $\zeta_{A} \otimes \zeta_{A}$ and $\mathrm{O}_{Y_{A}}$.

Thanks to the lemma we see that the sheaf

$$
\mathrm{O}_{Y_{A}} \oplus \zeta_{A}
$$

has the structure of an $\mathrm{O}_{Y_{A}}$-algebra, so we have an associated double covering.

Definition 1.4. We denote by $X_{A}$ this double covering; the scheme $X_{A}$ is called a double EPW sextic. We denote by

$$
f_{A}: X_{A} \rightarrow Y_{A}
$$

the covering map.

The scheme $X_{A}$ is endowed with a polarization $h_{A}=f_{A}^{*} \mathcal{O}_{Y_{A}}(1)$.

Remark 1.5. The ramification locus of the map $f_{A}$ is $Y_{A}$ [2]. To see this we just need to observe that by construction the ramification locus is the locus where the sheaf $\zeta_{A}$, or equivalently the sheaf $\xi_{A}$, is not locally free. Since $i_{A *}\left(\xi_{A}\right)$ is the cokernel of the map

$$
\lambda_{A}: F \rightarrow \mathbb{O}_{\mathbb{P}(V)} \otimes A^{\vee},
$$

we see that the rank of $\xi_{A}$ jumps exactly along $Y_{A}[2]$, hence our claim. 
Definition 1.6 [O'Grady 2010]. We let

$$
\Sigma=\left\{A \in \mathbb{L} \mathbb{G}\left(\bigwedge^{3} V\right) \mid \bigwedge^{3}(W) \subset A \text { for some } W \subset V, \operatorname{dim} W=3\right\} .
$$

In other words, $\Sigma$ is the set of Lagrangian subspaces of $\bigwedge^{3} V$ containing a decomposable form. We also let

$$
\Delta=\left\{A \in \mathbb{L} \mathbb{G}\left(\bigwedge^{3} V\right) \mid Y_{A}[3] \neq \varnothing\right\} \subset \mathbb{L} \mathbb{G}\left(\bigwedge^{3} V\right) .
$$

Finally we define

$$
\mathbb{L} \mathbb{G}\left(\bigwedge^{3} V\right)^{0}=\mathbb{L} \mathbb{G}\left(\bigwedge^{3} V\right) \backslash(\Sigma \cup \Delta) .
$$

We recall the following characterization of smooth double EPW sextics:

Theorem 1.7 [O'Grady 2010]. The double covering $X_{A}$ is smooth if and only if

$$
A \in \mathbb{L} \mathbb{G}\left(\bigwedge^{3} V\right)^{0} .
$$

The relevance of these double coverings stems from the following result.

Theorem 1.8 [O'Grady 2010]. Let $A \in \mathbb{L} \mathbb{G}\left(\bigwedge^{3} V\right)^{0}$. Then $X_{A}$ is an irreducible symplectic variety. The Beauville-Bogomolov form on $H^{2}(X, \mathbb{Z})$ is the same as that of $S^{[2]}$, where $S$ is a $K 3$ surface, and the Fujiki constant of $X_{A}$ is 3.

Let $Z_{A}=f_{A}^{-1}\left(Y_{A}[2]\right)$; this is the branch locus for the 2:1 covering, hence it is isomorphic to $Y_{A}$ [2] itself. Since the covering involution is antisymplectic, the symplectic form restricts to 0 on $Z_{A}$, that is, $Z_{A}$ is isotropic. Under mild assumptions $Z_{A}$ is a surface, hence a Lagrangian surface inside $X_{A}$. More precisely:

Proposition 1.9 [O'Grady 2010]. Let $A \in \mathbb{L} \mathbb{G}\left(\bigwedge^{3} V\right)^{0}$. Then $Y_{A}$ [2] is a smooth connected surface of degree 40, with $\chi_{\mathrm{top}}\left(Y_{A}[2]\right)=192$.

We will need the following relation in the Chow group. It appears in [O'Grady 2008b, Proposition 4.9], with a different proof from the one given below.

Proposition 1.10. Let $A \in \mathbb{L} \mathbb{G}\left(\bigwedge^{3} V\right)^{0}, Z=Z_{A}$. The canonical class of $Z$ satisfies

$$
2 K_{Z}=\mathrm{O}_{Z}(6)
$$

in $C H^{*}(Z)$.

Remark 1.11. The proposition determines $K_{Z}$ only up to 2-torsion. Namely we can rewrite it as

$$
K_{Z}=\mathrm{O}_{Z}(3)+\kappa,
$$

where $\kappa$ is a 2-torsion class. One can use the deformation argument in [Ferretti 2012] and the results of [Welters 1981] to show that the class $\kappa$ is really nonzero. 
Proof. For simplicity let $W=f(Z)$ denote the singular set of $Y$. We know that on $W$ the map $\lambda$ has constant rank 8 , so we get the following exact sequence of vector bundles on $W$ :

$$
\left.0 \longrightarrow \mathscr{K} \longrightarrow F \stackrel{\left.\lambda\right|_{W}}{\longrightarrow} \mathrm{O}_{W} \otimes\left(\bigwedge^{3} V / A\right) \longrightarrow \zeta\right|_{W} \longrightarrow 0 .
$$

Here $\mathscr{K}$ is defined to be the kernel of $\left.\lambda\right|_{W}$; it has rank 2 . Identifying $W$ with its preimage $Z \subset X$, we claim that the following isomorphisms hold:

$$
\begin{aligned}
\zeta{ }_{W} & \cong \mathcal{N}_{Z / X}, \\
\mathcal{K} & \cong \mathcal{N}_{Z / X}^{\vee} .
\end{aligned}
$$

Assuming (1-6) and (1-7) for a moment, the exact sequence in (1-5) gives

$$
c_{1}\left(\mathcal{N}_{Z / X}^{\vee}\right)-c_{1}(F)-c_{1}\left(\mathcal{N}_{Z / X}\right)=0,
$$

hence

$$
2 c_{1}\left(\mathcal{N}_{Z / X}\right)=-c_{1}(F)=\mathfrak{O}_{Z}(6) .
$$

Since $X$ has trivial canonical class, it follows that

$$
2 K_{Z}=2 c_{1}\left(\mathcal{N}_{Z / X}\right)=\mathcal{O}_{Z}(6),
$$

as desired.

So we now turn to the proof of (1-6) and (1-7). Let $p \in Z$; then the covering involution $\varphi$ fixes $p$, so $\varphi^{*}$ acts on $T_{p} X$. This gives a decomposition

$$
T_{p} X=\left(T_{p} X\right)_{+} \oplus\left(T_{p} X\right)_{-}
$$

in eigenspaces for $\varphi^{*}$, with eigenvalues \pm 1 . Since $Z$ is the fixed locus of $\varphi$,

$$
\left(T_{p} X\right)_{+}=T_{p} Z
$$

On the other hand, since

$$
X=\operatorname{Spec}\left(\mathscr{O}_{Y} \oplus \zeta\right)
$$

we can identify

$$
\left(T_{p} X\right)_{-} \cong \zeta_{f(p)}
$$

It follows that

$$
\left(\mathcal{N}_{Z / X}\right)_{p} \cong \zeta_{f(p)}
$$

this fiber-wise identification is easily seen to globalize, hence yielding the isomorphism in (1-6).

For the other, we show that $\left.\mathscr{K} \cong \zeta\right|_{W} ^{\vee}$. Indeed observe that over $W$ we have

$$
\mathscr{K}_{v}=F_{v} \cap A \quad \text { and } \quad \zeta_{v}=\bigwedge^{3} V /\left(F_{v}+A\right) .
$$


The symplectic form identifies $\mathcal{K}_{v}^{\vee}$ with the quotient $\bigwedge^{3} V /\left(F_{v} \cap A\right)^{\perp}$, and since both $A$ and $F_{v}$ are Lagrangian we have

$$
\left(F_{v} \cap A\right)^{\perp}=F_{v}^{\perp}+A^{\perp}=F_{v}+A,
$$

thereby proving the isomorphism (1-7).

Corollary 1.12. For $A \in \mathbb{L} \mathbb{G}\left(\bigwedge^{3} V\right)^{0}$ the surface $Z_{A} \cong Y_{A}$ [2] is of general type.

Plan of the paper. Before turning to the proof of Theorem 1.1, we explain the overall plan. Let $X=X_{A}$ be a smooth double EPW sextic, and denote its polarization $h=h_{A}$. The symplectic form gives an isomorphism

$$
T_{X} \cong \Omega_{X}^{1},
$$

hence the odd Chern classes vanish. So we only need to consider $c_{2}(X)$ and $c_{4}(X)$. Moreover if $A$ is very general in $\mathbb{L} \mathbb{G}\left(\bigwedge^{3} V\right)$, the group $\operatorname{Pic}\left(X_{A}\right)$ is cyclic, generated by $h_{A}$, so the second conclusion of Theorem 1.1 follows from the first.

The only relations in cohomology can be in degree 4,6 , or 8 . Lemma 2.3 excludes the existence of relations of degree 4 , hence we are left with relations in degree 6 or 8; these are listed in Propositions 2.1 and 2.2.

Since $h^{4}, c_{2}(X) \cdot h, c_{2}(X)^{2}$, and $c_{4}(X)$ are all proportional in cohomology, there must be some distinguished 0 -cycle $\theta$ on $X$, such that all these classes are multiples of $\theta$ in $\mathrm{CH}^{4}(X)$. We shall define $\theta$ as the class of any point on a suitable surface inside $X_{A}$; actually it will be easier to work with $Y_{A}$ and pull back everything to $X_{A}$ later.

Hence we look for a surface $S \subset X$ such that $C H^{2}(S)$ is trivial, so each point on $S$ is rationally equivalent to each other. For instance, in the proof of the conjecture in the case where $X$ is the Fano variety of a cubic fourfold, Voisin [2008] used a rational surface. In that case there is a family of Lagrangian surfaces on $X$, which are simply the Fano varieties of hyperplane sections of the cubic; if the section is singular enough, its Fano variety turns out to be rational.

In our case this construction is a delicate point: the analogue of $S$ is an Enriques surface, but exhibiting it is complicated. The construction that we need is provided in [Ferretti 2012].

So the plan is as follows. In Section 2 we carry out the cohomology computations on $X$. In Section 3 we use the surface constructed in [Ferretti 2012] to define the class $\theta$. In the rest of the paper we find enough relations in the Chow ring to finish the proof of the main theorem.

\section{Cohomology computations}

Let $X=X_{A}$ be a smooth double EPW sextic. In this section we compute the cohomological invariants of $X$, partly following [O'Grady 2008b]. We shall find 
all relations in cohomology between $h$ and the Chern classes of $X$. In later sections we shall show that these relations hold in the Chow ring.

Let $\sigma$ be the symplectic form on $X$. Since the canonical of $X$ is trivial

$$
H^{4,0}(X)=H^{0}\left(X, \Omega_{X}^{4}\right)
$$

is generated by $\sigma^{2}$. Moreover it is known that $H^{3}(X)=0$, so we can compute the Euler characteristic

$$
\chi\left(X, \mathscr{O}_{X}\right)=h^{0,0}(X)+h^{2,0}(X)+h^{4,0}(X)=3 .
$$

The symplectic form on $X$ gives an isomorphism

$$
T_{X} \cong \Omega_{X}^{1},
$$

hence the odd Chern classes vanish. The Hirzebruch-Riemann-Roch theorem for $X$ simplifies to

$$
3=\chi\left(X, \mathrm{O}_{X}\right)=\frac{1}{240}\left(c_{2}(X)^{2}-\frac{1}{3} c_{4}(X)\right) .
$$

We introduce some more notation. Let us call

$$
q \in \operatorname{Sym}^{2}\left(H^{2}(X, \mathbb{Q})^{\vee}\right)
$$

the Beauville-Bogomolov form of $X$. Since it is nondegenerate, it allows us to give an identification

$$
H^{2}(X, \mathbb{Q}) \cong H^{2}(X, \mathbb{Q})^{\vee}
$$

hence we obtain a dual quadratic form

$$
q^{\vee} \in \operatorname{Sym}^{2}\left(H^{2}(X, \mathbb{Q})\right) .
$$

From [Verbitsky 1996, Theorem 1.5] (together with the computation of the Betti numbers in [Göttsche 1990]) we know that the cup product yields an isomorphism between $\operatorname{Sym}^{2}\left(H^{2}(X, \mathbb{Q})\right)$ and $H^{4}(X, \mathbb{Q})$, so we can regard $q^{\vee}$ as an element of $H^{4}(X, \mathbb{Q})$.

O'Grady [2008b] proves that we have the relation

$$
q^{\vee}=\frac{5}{6} c_{2}(X),
$$

and that for any $\alpha, \beta \in H^{2}(X, \mathbb{Q})$ we have

$$
q^{\vee} \cdot \alpha \cdot \beta=25 q(\alpha, \beta) .
$$

We now work out the relations in the cohomology of $X$. Let

$$
h=c_{1}\left(f^{*} \mathscr{O}_{Y}(1)\right) \in H^{2}(X) .
$$


Proposition 2.1. In the cohomology ring $H^{*}(X, \mathbb{Q})$ we have

$$
h^{4}=12, \quad h^{2} \cdot c_{2}(X)=60, \quad c_{2}(X)^{2}=828, \quad c_{4}(X)=324 .
$$

Proof. The first and the last relations are easily handled. Indeed,

$$
h^{4}=2 \operatorname{deg}(Y)=12 .
$$

As for the last one we have

$$
c_{4}(X)=\chi(X)
$$

and since $X$ is a deformation of $S^{[2]}$, where $S$ is a K3, we have

$$
\chi(X)=\chi\left(S^{[2]}\right)=324 \text {. }
$$

By O'Grady's computations (2-3) and (2-2) we also have

$$
c_{2}(X) \cdot h^{2}=\frac{6}{5} q^{\vee} \cdot h^{2}=\frac{25 \cdot 6}{5} q(h, h)=60 .
$$

Finally we can use (2-1) to obtain $c_{2}(X)^{2}=828$.

In degree 6 the only possible relation is a linear dependency between $h^{3}$ and $c_{2}(X) \cdot h$, and indeed we have:

Proposition 2.2. There is a relation

$$
c_{2}(X) \cdot h=5 h^{3}
$$

$H^{6}(X, \mathbb{Q})$.

Proof. From O'Grady's relation (2-3) we get

$$
6 q^{\vee} \cdot h \cdot \alpha=6 \cdot 25 q(h, \alpha)
$$

for all $\alpha \in H^{2}(X)$. On the other hand, by polarization of Fujiki's relation we obtain

$$
25 h^{3} \cdot \alpha=25 \cdot 3 \cdot q(h, h) q(h, \alpha)=6 \cdot 25 q(h, \alpha) .
$$

So Poincaré duality implies that

$$
25 h^{3}=6 q^{\vee} \cdot h
$$

modulo torsion, and using (2-2) we get the thesis.

We can instead exclude relations in degree 4:

Lemma 2.3. The classes $h^{2}$ and $c_{2}(X)$ are linearly independent inside $H^{2}(X)$.

This fact appears inside the proof of [O'Grady 2008b, Claim 3.1], but we add a short proof for completeness. 
Proof. We can substitute $c_{2}(X)$ with its multiple $q^{\vee}$. Assume that we have a relation

$$
h^{2}+\lambda q^{\vee}=0
$$

for some $\lambda \in \mathbb{C}$. Then we get

$$
h^{2} \alpha^{2}=-25 \lambda q(\alpha, \alpha)
$$

for all $\alpha \in H^{2}(X)$. By polarization of the Fujiki formula we also obtain

$$
h^{2} \alpha^{2}=q(\alpha, \alpha) q(h, h)+2 q(h, \alpha)^{2} .
$$

So if $q(\alpha, \alpha)=0$ we obtain $q(h, \alpha)=0$. This means that $q$ is degenerate (the quadric defined by $q$ would be contained in a hyperplane of $\mathbb{P} H^{2}(X)$ ), which is a contradiction.

Finally, it will be useful to write out the explicit form of Hirzebruch-RiemannRoch, using the above computations for the characteristic classes of $X$. We let

$$
\mathrm{O}_{X}(1)=f^{*} \mathrm{O}_{Y}(1) \text {. }
$$

Then $\mathscr{O}_{X}(n)$ is ample on $X$, and since $K_{X}$ is trivial, Kodaira vanishing yields

$$
\chi\left(X, O_{X}(n)\right)=h^{0}\left(X, O_{X}(n)\right) .
$$

The formula of Hirzebruch-Riemann-Roch then reads

$$
h^{0}\left(X, \mathcal{O}_{X}(n)\right)=\frac{h^{4}}{24} n^{4}+\frac{c_{2}(X) \cdot h^{2}}{24} n^{2}+\chi\left(\mathbb{O}_{X}\right)=\frac{1}{2} n^{4}+\frac{5}{2} n^{2}+3 .
$$

\section{Definition of the class $\theta$}

Let $X=X_{A}$ be a double EPW sextic, $f: X \rightarrow Y$ the double covering. Our first task is to define a class

$$
\theta \in C H^{4}(X)
$$

of degree 1 . Then we will show that the relations

$$
h^{4}=12 \theta, \quad h^{2} c_{2}(X)=60 \theta, \quad c_{2}(X)^{2}=828 \theta, \quad c_{4}(X)=324 \theta
$$

hold.

It will actually be easier to work on $Y$, so we should find the relationship between $\mathrm{CH}(\mathrm{X})$ and $\mathrm{CH}(\mathrm{Y})$.

Remark 3.1. The map $f: X \rightarrow Y$ induces a push-forward morphism

$$
f_{*}: C H(X) \rightarrow C H(Y),
$$

because $f$ is proper (for the construction of Chow rings and morphisms between them see [Fulton 1984, Chapter 1]). On the other hand $f^{*}$ is usually defined for flat 
maps with fibers of constant dimension, or when the target is smooth, and neither is the case.

Following [Fulton 1984, Example 1.7.6] we can define $f^{*}$ in our situation. Indeed, Fulton shows that if

$$
Y=X / G
$$

is the quotient of $X$ by the action of a finite group $G$, we have a canonical isomorphism

$$
C H(Y)_{\mathbb{Q}} \cong C H(X)_{\mathbb{Q}}^{G},
$$

where as usual $C H(Y)_{\mathbb{Q}}=C H(Y) \otimes \mathbb{Q}$. So if $f$ is the quotient map we can define $f^{*}$ by the composition

$$
C H(Y)_{\mathbb{Q}} \stackrel{\cong}{\rightarrow} C H(X)_{\mathbb{Q}}^{G} \hookrightarrow C H(X)_{\mathbb{Q}} .
$$

Fulton also shows that the composition

$$
C H(Y)_{\mathbb{Q}} \stackrel{f^{*}}{\rightarrow} C H(X)_{\mathbb{Q}} \stackrel{f_{*}}{\rightarrow} C H(Y)_{\mathbb{Q}}
$$

is the multiplication map by $\sharp G$.

In our situation $G=\langle\varphi\rangle$, where $\varphi$ is the covering involution, and the composition above is multiplication by 2 .

Moreover we observe that

$$
C H(Y)_{\mathbb{Q}} \cong C H(X)_{\mathbb{Q}}^{\mathbb{Z} / 2 \mathbb{Z}}
$$

is a subring of $C H(X)_{\mathbb{Q}}$, so we can multiply cycle classes on $Y$ even if it is singular.

We now recall the main result in [Ferretti 2012]. There we produce a surface $S \subset Y$ such that $C H^{2}(S)=\mathbb{Z}$; this will be the starting point for our investigation of $C H^{*}(Y)$. Let us fix a Lagrangian subspace $A \in \mathbb{L} \mathbb{G}\left(\bigwedge^{3} V\right)$. If $B$ is another Lagrangian subspace such that

$$
\operatorname{dim}(A \cap B)=9,
$$

it follows from the Grassmann formula that $Y_{B}[2] \subset Y_{A}$.

Theorem [Ferretti 2012]. Let $A$ be a Lagrangian subspace in $\mathbb{L} \mathbb{G}\left(\bigwedge^{3} V\right)^{0}$. Then there exists another Lagrangian subspace $B$ such that $\operatorname{dim}(A \cap B) \geq 9$, and such that $S:=Y_{B}$ [2] is either a (singular) Enriques surface, or a degeneration of such surfaces. In either case we have

$$
C H^{2}(S) \cong \mathbb{Z} .
$$

Moreover we have the relation

$$
[S]=\left[Y_{A}[2]\right]
$$


in $C H^{2}\left(X_{A}\right)$.

So for such a choice of $B$ we know that all points on $S=Y_{B}$ [2] are rationally equivalent. We define $\bar{\theta}$ as the class of any such point. We need to do some checks in order to show that this is actually well-defined. We also define

$$
\theta=\frac{1}{2} f^{*}(\bar{\theta}) \in C H^{4}\left(X_{A}\right)_{\mathbb{Q}} .
$$

Lemma 3.2. Let $B, B^{\prime} \in \mathbb{L} \mathbb{G}\left(\bigwedge^{3} V\right)$ such that (3-1) holds. Then

$$
Y_{B}[2] \cap Y_{B^{\prime}}[2] \neq \varnothing \text {. }
$$

Proof. It is enough to show that

$$
Y_{B}[2] \cdot Y_{B^{\prime}}[2] \neq 0
$$

in $C H^{*}\left(Y_{A}\right)$. Thanks to (3-2) it will be enough to prove that

$$
Y_{A}[2]^{2} \neq 0
$$

By the definition of the ring structure on $C H^{*}\left(Y_{A}\right)$ we need to prove that

$$
Z_{A}^{2} \neq 0 \text { in } C H^{*}\left(X_{A}\right) \text {. }
$$

But actually $Z_{A}^{2} \neq 0$ already in cohomology. Indeed, using the fact that $Z_{A}$ is Lagrangian, we have

$$
Z_{A}^{2}=c_{2}\left(\mathcal{N}_{Z_{A} / X_{A}}\right)=c_{2}\left(\Omega_{Z_{A}}^{1}\right)=c_{2}\left(Z_{A}\right)=\chi_{\text {top }}\left(Z_{A}\right)=192
$$

by Proposition 1.9 .

By the previous lemma we see that the class of $\bar{\theta} \in C H^{4}\left(Y_{A}\right)$ is actually independent of the chosen Lagrangian subspace $B$ such that (3-1) holds.

\section{Relations coming from vector bundle morphisms}

In this section we omit $A$ from the notation, for clarity. Fix a Lagrangian subspace $A \in \mathbb{L} \mathbb{G}\left(\bigwedge^{3} V\right)^{0}$ and denote $X=X_{A}, Y=Y_{A}$, and $Z=Z_{A}$. We shall exhibit a number of relations coming from exact sequences of sheaves on $X$ and $Y$.

Lemma 4.1. The following relation holds in $C H(X)$ :

$$
3 Z=15 h^{2}-c_{2}(X)
$$

Proof. We consider $f$ as a map $X \rightarrow \mathbb{P}^{5}$, so it induces a morphism of vector bundles over $X$ :

$$
d f: T_{X} \rightarrow f^{*} T_{\mathbb{P} 5}
$$


We notice that $d f$ is injective outside $Z$, so we can see $Z$ as a degeneracy locus for this morphism. We then apply the Thom-Porteous formula in the form stated in [Fulton 1984, §14.4]. In their notation we have $e=4, f=5$, and $k=3$.

This yields a cycle class

$$
\mathbb{D}_{3}(d f) \in C H^{2}(Z)
$$

whose support is $Z$, and such that the image of $\mathbb{D}_{3}(d f)$ in $C H^{2}(X)$ is

$$
\Delta_{2}^{(1)}\left(c\left(f^{*} T_{\mathbb{P}^{5}}-T_{X}\right)\right)=c_{2}\left(f^{*} T_{\mathbb{P}^{5}}-T_{X}\right) .
$$

Here the total Chern class

$$
c\left(f^{*} T_{\mathbb{P} 5}-T_{X}\right)
$$

is defined formally in such a way that Whitney's formula holds, that is,

$$
c\left(T_{X}\right) \cdot c\left(f^{*} T_{\mathbb{P} 5}-T_{X}\right)=c\left(f^{*} T_{\mathbb{P} 5}\right) .
$$

From the last equation and the fact that $c_{1}\left(T_{X}\right)=0$ (since $X$ is symplectic) we can obtain

$$
c_{2}\left(f^{*} T_{\mathbb{P}^{5}}-T_{X}\right)=f^{*} c_{2}\left(T_{\mathbb{P} 5}\right)-c_{2}\left(T_{X}\right)=15 h^{2}-c_{2}(X) .
$$

Since $\mathbb{D}_{3}(d f)$ has support on $Z$, which is irreducible, we find that

$$
k Z=15 h^{2}-c_{2}(X)
$$

for some $k \in \mathbb{Z}$. To find the right $k$, we observe that, again by [Fulton 1984, Theorem 14.4(c)], we have

$$
\mathbb{D}_{3}(d f)=\left[D_{3}(d f)\right],
$$

where $D_{3}(d f)$ is the degeneracy locus of $d f$. In other words $D_{3}(d f)$ is just $Z$, with the scheme structure given by the vanishing of all $4 \times 4$ minors of $d f$.

The map

$$
f: X \rightarrow Y \subset \mathbb{P}^{5}
$$

has, in suitable analytic coordinates around a point of $Z$, the local form

$$
f(x, y, z, t) \underset{\text { loc }}{=}\left(x^{2}, x y, y^{2}, z, t\right) .
$$

The differential of $f$ is then

$$
d f \underset{\operatorname{loc}}{=}\left(\begin{array}{cccc}
2 x & 0 & 0 & 0 \\
y & x & 0 & 0 \\
0 & 2 y & 0 & 0 \\
0 & 0 & 1 & 0 \\
0 & 0 & 0 & 1
\end{array}\right)
$$


equating to 0 the determinants of its $3 \times 3$ minors yields

$$
D_{3}(d f) \underset{\text { loc }}{=} V\left(x^{2}, x y, y^{2}\right) .
$$

So we see that $D_{3}(d f)$ has multiplicity 3 at each point of $Z$, hence $k=3$.

Alternatively we could multiply (4-1) by $h^{2}$ to find

$$
k Z \cdot h^{2}=15 h^{4}-c_{2}(X) \cdot h^{2} .
$$

If we look at this relation in cohomology it becomes, thanks to Proposition 2.1,

$$
40 k=15 \cdot 12-60,
$$

so $k=3$.

We take a closer look at the differential of

$$
f: X \rightarrow \mathbb{P}^{5} .
$$

As a map of vector bundles, this is not injective exactly on $Z$. Hence it is always injective on stalks; in other words

$$
d f: T_{X} \rightarrow f^{*} T_{\mathbb{P} 5}
$$

is an injective map of sheaves. Let $\mathscr{R}$ denote its cokernel; this is locally free of rank 1 outside $Z$. So we have the exact sequence

$$
0 \longrightarrow T_{X} \longrightarrow f^{*} T_{\mathbb{P} 5} \longrightarrow \mathscr{R} \longrightarrow 0 .
$$

We now dualize it applying $\mathscr{H} o m\left(\cdot, O_{X}\right)$. We remark that

$$
\operatorname{Hom}\left(\mathscr{R}, O_{X}\right)
$$

is torsion-free, of rank one, and one can check in local coordinates that it is a line bundle. By (4-2) we get $c_{1}(\mathscr{R})=6 h$, hence

$$
\operatorname{Hom}\left(\Re, O_{X}\right) \cong O_{X}(-6) .
$$

Then we note that

$$
\mathscr{E} x t^{1}\left(f^{*}\left(T_{\mathbb{P}^{5}}\right), \mathscr{O}_{X}\right)=0,
$$

because both sheaves are locally free. So if we let

$$
2=\mathscr{E} x t^{1}\left(\mathscr{R}, \mathscr{O}_{X}\right),
$$

the dual of (4-2) becomes

$$
0 \longrightarrow \mathrm{O}_{X}(-6) \longrightarrow f^{*}\left(\Omega_{\mathbb{P}^{5}}^{1}\right) \stackrel{d f^{T}}{\longrightarrow} \Omega_{X}^{1} \longrightarrow 2 \longrightarrow 0 .
$$


We remark that 2 is set-theoretically supported on $Z$, because both $\mathscr{R}$ and $O_{X}$ are locally free outside $Z$. Actually the schematic support of 2 is $2 Z$, that is, the subscheme of $X$ defined by the ideal $\mathscr{I}_{Z}^{2}$. This follows from:

Lemma 4.2. Let 2 be as above; then $\operatorname{Ann}(2)=\Phi_{Z}^{2}$.

Proof. We only need to prove this locally. As in the proof of Lemma 4.1 we can choose local coordinates on $X$ such that

$$
f(x, y, z, t) \underset{\text { loc }}{=}\left(x^{2}, x y, y^{2}, z, t\right) ;
$$

then $d f^{T}$ has the matrix

$$
d f^{T} \underset{\operatorname{loc}}{=}\left(\begin{array}{ccccc}
2 x & y & 0 & 0 & 0 \\
0 & x & 2 y & 0 & 0 \\
0 & 0 & 0 & 1 & 0 \\
0 & 0 & 0 & 0 & 1
\end{array}\right)
$$

hence we have the presentation

$$
2 \underset{\operatorname{loc}}{=} \frac{\langle d x, d y\rangle}{\langle x d x, x d y+y d x, y d y\rangle} .
$$

A given $h(x, y) \in \mathbb{C}[x, y]$ then annihilates 2 if and only if both $h d x$ and $h d y$ belong to the $k[x, y]$-module generated by $x d x, x d y+y d x$, and $y d y$.

Let us make this more explicit. Assume that

$$
h(x, y) d x=a(x, y) x d x+b(x, y) \cdot(x d y+y d x)+c(x, y) y d y .
$$

This yields

$$
\begin{aligned}
h(x, y) & =x a(x, y)+y b(x, y), \\
0 & =x b(x, y)+y c(x, y) .
\end{aligned}
$$

The second equation implies $b(x, y)=y b^{\prime}(x, y)$, so the first becomes

$$
h(x, y)=x a(x, y)+y^{2} b^{\prime}(x, y) .
$$

If $h$ can be written this way, then we can choose $c$ so that the second condition is satisfied. In short

$$
h(x, y) d x \in\langle x d x, x d y+y d x, y d y\rangle_{k[x, y]}
$$

if and only if $h \in\left(x, y^{2}\right)$.

We have the symmetric condition for $h(x, y) d y$, so we conclude that $h \in \operatorname{Ann}(2)$ if and only if

$$
h \in\left(x, y^{2}\right) \cap\left(x^{2}, y\right)=\left(x^{2}, x y, y^{2}\right) .
$$


The last equality between ideals can be proved, for instance, by the remark that both $\left(x, y^{2}\right) \cap\left(x^{2}, y\right)$ and $\left(x^{2}, x y, y^{2}\right)$ consist of the polynomials $h$ such that

$$
h(0,0)=\frac{\partial h}{\partial x}(0,0)=\frac{\partial h}{\partial y}(0,0)=0 .
$$

Finally, $\left(x^{2}, x y, y^{2}\right)$ is exactly the square of the ideal $(x, y)$ which locally defines $Z$.

We now produce another exact sequence involving 2. Let

$$
i: Z \hookrightarrow X
$$

denote the inclusion. Recall that we have a canonical identification

$$
\Phi_{Z} / \mathscr{I}_{Z}^{2} \cong i_{*} \mathcal{N}_{Z / X}^{\vee}:
$$

locally the function $g$ vanishing on $Z$ corresponds to the normal covector $d g$. Consider the natural projection

$$
\pi:\left.\Omega_{X}^{1}\right|_{Z} \rightarrow \mathcal{N}_{Z / X}^{\vee}
$$

we see this as a map on $X$ :

$$
\pi: \Omega_{X}^{1} \rightarrow \Phi_{Z} / \mathscr{I}_{Z}^{2}
$$

Lemma 4.3. We have $\pi \circ d f^{T}=0$.

Proof. We keep the notation of the proof of Lemma 4.2. We need only to verify the thesis on $Z$. The image of $d f^{T}$ is generated by

$$
x d x, x d y+y d x, y d y, d z, d t .
$$

The first three elements vanish on $Z$, while the latter two are in the kernel of $\pi$.

The above lemma and the exact sequence in (4-3) provide us a surjective map

$$
\alpha: 2 \rightarrow i_{*}\left(\mathcal{N}_{Z / X}^{\vee}\right)
$$

Lemma 4.4. The kernel of $\alpha$ is $i_{*}\left(\operatorname{det} T_{Z}\right)$.

Proof. We can see this explicitly in local coordinates. Keeping the notation of the above proofs, 2 is locally generated, on $Z$, by $d x, d y$, and $x d y=-y d x$. The conormal bundle $\mathcal{N}_{Z / X}^{\vee}$ is generated by $d x$ and $d y$, and $\alpha$ is the obvious projection.

The kernel of $\alpha$ is then generated by $x d y$. Under the identification in (4-4) this corresponds to the generator $d x \wedge d y$ of $\wedge^{2} \mathcal{N}_{Z / X}^{\vee}$.

So

$$
\operatorname{ker} \alpha=i_{*}\left(\operatorname{det} \mathcal{N}_{Z / X}^{\vee}\right) \cong i_{*}\left(\operatorname{det} T_{Z}\right)
$$

since $Z$ is Lagrangian. 
Thanks to the lemma we get the exact sequence we are looking for:

$$
0 \longrightarrow i_{*}\left(\operatorname{det} T_{Z}\right) \longrightarrow 2 \longrightarrow i_{*} T_{Z} \longrightarrow 0 .
$$

We can now find new relations in the Chow ring of $X$.

Proposition 4.5. In $\mathrm{CH}(X)_{\mathbb{Q}}$ we have

$$
c_{2}(X) \cdot h=5 h^{3}
$$

and $c_{4}(X)$ is a linear combination of $h^{4}, c_{2}(X) \cdot h^{2}$, and $c_{2}(X)^{2}$.

Proof. This is just a matter of putting together the relations that come from the exact sequences (4-3) and (4-5).

We start from (4-3), which yields

$$
(1-6 h) \cdot\left(1+c_{2}(X)+c_{4}(X)\right)=(1-h)^{6} \cdot\left(1+c_{1}(2)+c_{2}(2)+c_{3}(2)+c_{4}(2)\right) .
$$

Comparing the terms in degree up to 2 we get:

$$
c_{1}(2)=0, \quad c_{2}(2)=c_{2}(X)-15 h^{2}=-3 Z,
$$

where the last equality is Lemma 4.1 . Then in degree 3 we have

$$
c_{3}(2)=6 h\left(c_{2}(2)-c_{2}(X)\right)+20 h^{3}=6 h \cdot\left(-15 h^{2}\right)+20 h^{3}=-70 h^{3},
$$

using the second of (4-6). Finally in degree 4 we get, using (4-6) and (4-7),

$c_{4}(X)=15 h^{4}+15 h^{2} \cdot c_{2}(2)-6 h \cdot c_{3}(2)+c_{4}(2)=15 h^{4}-45 h^{2} \cdot Z+420 h^{4}+c_{4}(2)$, hence

$$
c_{4}(2)=c_{4}(X)-435 h^{4}+45 h^{2} \cdot Z .
$$

Next we look at the relations coming from (4-5). To do this we shall use Grothendieck-Riemann-Roch, which for the closed embedding

$$
i: Z \hookrightarrow X
$$

takes the form

$$
\operatorname{ch}\left(i_{*} \mathscr{F}\right)=i_{*}\left(\operatorname{ch}(\mathscr{F}) \cdot \operatorname{td}\left(\mathcal{N}_{Z / X}\right)^{-1}\right),
$$

for any $\mathscr{F} \in \operatorname{Coh}(Z)$. This is because in our situation we have

$$
R^{k} i_{*}(\mathscr{F})=0
$$

for all such $\mathscr{F}$, thanks to [Hartshorne 1977, Corollary III.11.2].

Using that $Z$ is Lagrangian we have $\mathcal{N}_{Z / X} \cong \Omega_{Z}^{1}$, so we can compute

$$
\begin{aligned}
\operatorname{td}\left(\mathcal{N}_{Z / X}\right) & =1-\frac{1}{2} c_{1}(Z)+\frac{1}{12}\left(c_{1}(Z)^{2}+c_{2}(Z)\right), \\
\operatorname{td}\left(\mathcal{N}_{Z / X}\right)^{-1} & =1+\frac{1}{2} c_{1}(Z)+\frac{1}{6} c_{1}(Z)^{2}-\frac{1}{12} c_{2}(Z) .
\end{aligned}
$$


Then we have

$$
\begin{aligned}
\operatorname{ch}\left(\operatorname{det} T_{Z}\right) & =1+c_{1}(Z)+\frac{1}{2} c_{1}(Z)^{2}, \\
\operatorname{ch}\left(T_{Z}\right) & =2+c_{1}(Z)+\frac{1}{2}\left(c_{1}(Z)^{2}-c_{2}(Z)\right) .
\end{aligned}
$$

So Grothendieck-Riemann-Roch for these sheaves becomes

$$
\begin{aligned}
\operatorname{ch}\left(i_{*} \operatorname{det} T_{Z}\right) & =i_{*}\left(1+\frac{3}{2} c_{1}(Z)+\frac{7}{6} c_{1}(Z)^{2}-\frac{1}{12} c_{2}(Z)\right), \\
\operatorname{ch}\left(i_{*} T_{Z}\right) & =i_{*}\left(2+2 c_{1}(Z)+\frac{4}{3} c_{1}(Z)^{2}-\frac{7}{6} c_{2}(Z)\right) .
\end{aligned}
$$

Next we use the fact that in $C H(Z)_{\mathbb{Q}}$ we have

$$
c_{1}(Z)=-K_{Z}=-3 i^{*}(h),
$$

thanks to Proposition 1.10. So we obtain

$$
\begin{aligned}
\operatorname{ch}\left(i_{*} \operatorname{det} T_{Z}\right) & =Z-\frac{9}{2} h \cdot Z+\frac{21}{2} h^{2} \cdot Z-\frac{1}{12} Z^{2}, \\
\operatorname{ch}\left(i_{*} T_{Z}\right) & =2 Z-6 h \cdot Z+12 h^{2} \cdot Z-\frac{7}{6} Z^{2} .
\end{aligned}
$$

We can use this to recover the Chern classes of $i_{*}\left(\operatorname{det} T_{Z}\right)$ and $i_{*}\left(T_{Z}\right)$. These are:

$$
\begin{array}{ll}
c_{1}\left(i_{*} \operatorname{det} T_{Z}\right)=0, & c_{2}\left(i_{*} \operatorname{det} T_{Z}\right)=-Z, \\
c_{3}\left(i_{*} \operatorname{det} T_{Z}\right)=-9 h \cdot Z, & c_{4}\left(i_{*} \operatorname{det} T_{Z}\right)=Z^{2}-63 h^{2} \cdot Z,
\end{array}
$$

and

$$
\begin{array}{ll}
c_{1}\left(i_{*} T_{Z}\right)=0, & c_{2}\left(i_{*} T_{Z}\right)=-2 Z, \\
c_{3}\left(i_{*} T_{Z}\right)=-12 h \cdot Z, & c_{4}\left(i_{*} T_{Z}\right)=9 Z^{2}-72 h^{2} \cdot Z .
\end{array}
$$

Finally we use the exact sequence (4-5) to get the Chern classes of 2. The first two are

$$
c_{1}(2)=0, \quad c_{2}(2)=-3 Z,
$$

in accordance with (4-6). Then we get

$$
c_{3}(2)=-21 h \cdot Z,
$$

and comparing with (4-7) we obtain

$$
-3 h \cdot Z=-10 h^{3} .
$$

Using Lemma 4.1 this is equivalent to

$$
c_{2}(X) \cdot h=5 h^{3} .
$$

Finally we get

$$
c_{4}(2)=12 Z^{2}-135 h^{2} \cdot Z ;
$$


comparing with (4-8) this yields

$$
12 Z^{2}-135 h^{2} \cdot Z=c_{4}(X)-435 h^{4}+45 h^{2} \cdot Z,
$$

and using again Lemma 4.1 to write $Z$ as a rational combination of $c_{2}(X)$ and $h^{2}$, we get the second claim of the thesis.

\section{Conclusion of the proof}

First we recall that we have defined the class

$$
\theta=\frac{1}{2} f^{*}(\bar{\theta})
$$

Here $\bar{\theta}$ is the class of any point on a surface $S \subset Y_{A}$. By (3-2) we know that

$$
\left[Y_{A}[2]\right]=[S] \text { in } C^{2}\left(Y_{A}\right) \text {. }
$$

We also let $\bar{h}=\mathscr{O}_{Y}(1)$, so that $h=f^{*}(\bar{h})$.

Lemma 5.1. There exists a line $L_{0} \subset Y$ which meets $Y_{B}[2]$.

Proof. Let $V$ be the union of lines contained in $Y$.

Step 1: $\operatorname{dim} V \geq 2$. Let $R \subset \operatorname{Gr}(2, V)$ be the locus of lines $\ell \subset Y_{A}$. We can obtain $R$ as follows. Let

$$
Y_{A}=V(g),
$$

where $g$ is a degree- 6 polynomial, and let $\mathscr{S}$ be the tautological subbundle on $\operatorname{Gr}(2, V)$, so that $\operatorname{Sym}^{6}\left(\mathscr{G}^{\vee}\right)$ is the fiber bundle whose fiber at $\ell$ is the vector space of homogeneous polynomials of degree 6 on $\ell$.

Then we can define a section

$$
s \in H^{0}\left(\operatorname{Gr}(2, V), \operatorname{Sym}^{6}\left(\mathscr{S}^{\vee}\right)\right)
$$

by the condition

$$
s(\ell)=\left.g\right|_{\ell} .
$$

By definition $R$ is the zero locus of $s$. It follows that

$$
\operatorname{dim} R \geq \operatorname{dim} \operatorname{Gr}(2, V)-\operatorname{rk} \operatorname{Sym}^{6}\left(\mathscr{Y}^{\vee}\right)=8-7=1,
$$

provided $R$ is not empty. But we can show that $R \neq \varnothing$ by computing the fundamental class

$$
[R]=c_{7}\left(\operatorname{Sym}^{6}\left(\mathscr{Y}^{\vee}\right)\right)=432 \cdot 134 \sigma_{4,3} .
$$

Here the notation is that of Schubert calculus, see for instance [Griffiths and Harris 1978, §1.5].

Since $V=\bigcup_{\ell \in R} \ell$ is birational to a $\mathbb{P}^{1}$-bundle over $R$, it follows that $\operatorname{dim} V \geq 2$. 
Step 2: There exists $B^{\prime}$ such that $A \cap B=A \cap B^{\prime}$ and $Y_{B^{\prime}}[2]$ meets $V$. Let

$$
U=A \cap B .
$$

In [Ferretti 2012] we have shown that there exists a divisor $D_{U} \subset Y_{A}$ which is covered by the surfaces $\left[Y_{B^{\prime}}[2]\right]$ as $B^{\prime}$ ranges through the Lagrangian subspaces which satisfy

$$
A \cap B^{\prime} \supset U \text {. }
$$

In particular $D_{U}$ has dimension 3 ; since two varieties of dimensions 2 and 3 in $\mathbb{P}^{5}$ always meet, it follows that

$$
D_{U} \cap V \neq \varnothing .
$$

So there exists a Lagrangian subspace $B^{\prime}$ such that $B^{\prime} \cap A=U$ and

$$
Y_{B^{\prime}}[2] \cap V \neq \varnothing \text {. }
$$

Step 3: B meets $V$. We lift everything to $X_{A}$, which is smooth, so intersection theory applies. Let

$$
\widetilde{V}_{1}=f^{-1}(V) \quad \text { and } \quad \widetilde{V}_{2}=f^{-1}\left(Y_{B^{\prime}}[2]\right) .
$$

One easily sees that on $X$

$$
\widetilde{V}_{1} \cdot \widetilde{V}_{2} \neq 0
$$

Since $f^{-1}\left(Y_{B}[2]\right)$ and $\widetilde{V}_{2}$ have the same homology class, it follows that

$$
\widetilde{V}_{1} \cdot f^{-1}\left(Y_{B}[2]\right) \neq 0
$$

in particular $\widetilde{V}_{1}$ must meet $f^{-1}\left(Y_{B}[2]\right)$, and so

$$
V \cap Y_{B}[2] \neq \varnothing \text {. }
$$

Using Lemma 5.1 we can start proving that

$$
h^{4}=6 \theta \text { in } C H(X) \text {. }
$$

Indeed let $L_{0}$ be any line meeting $S$ and let $\Lambda$ be any plane containing $L_{0}$. Then $\bar{h}^{3}$ is represented by the intersection

$$
\Lambda \cdot Y=L_{0}+C,
$$

where $C$ is a quintic on $\Lambda$. Multiplying by $\bar{h}$ we obtain

$$
\bar{h}^{4}=L_{0} \cdot \bar{h}+C \cdot \bar{h} .
$$

We claim that this is represented by a 0 -cycle supported on $L_{0}$. This is clear for the first addend; for the second we represent $\bar{h}$ by a hyperplane containing $L_{0}$ and transverse to $\Lambda$. It follows that $C \cdot \bar{h}$ is supported on $C \cap L_{0}$. 
Since $L_{0}$ is rational, $C H^{1}\left(L_{0}\right) \cong \mathbb{Z}$, so $\bar{h}^{4}$ is rationally equivalent to a multiple of a point of $L_{0}$. Finally $L_{0} \cap S \neq \varnothing$, so we get

$$
\bar{h}^{4}=k \bar{\theta} \quad \text { in } C H^{4}(Y)_{\mathbb{Q}}
$$

for some $k \in \mathbb{Q}$.

Pulling back this relation to $X$ and using $f^{*}(\bar{h})=h, f^{*}(\bar{\theta})=2 \theta$ we see that $h^{4}=2 k \theta$ in $C H^{4}(X)_{\mathbb{Q}}$. Since in cohomology we have $h^{4}=12$ we must have $k=6$, and so (5-1) is proved.

Next we show that

$$
h^{2} \cdot c_{2}(X)=60 \bar{\theta} .
$$

We start from Lemma 4.1; pushing forward that relation we get

$$
3\left[Y_{A}[2]\right]=15 \cdot 4 \bar{h}^{2}-f_{*} c_{2}(X) \text { in } C H^{2}(Y) .
$$

Multiplying (5-3) by $\bar{h}^{2}$ we get

$$
\bar{h}^{2} \cdot f_{*} c_{2}(X)=60 \bar{h}^{4}-3 \bar{h}^{2} \cdot\left[Y_{A}[2]\right] .
$$

We already proved that $\bar{h}^{4}$ is a multiple of $\bar{\theta}$, and the cycle class

$$
\bar{h}^{2} \cdot\left[Y_{A}[2]\right]=\bar{h}^{2} \cdot[S]
$$

is supported on $S$, hence it is a rational multiple of $\bar{\theta}$ too.

We conclude that the relation (5-2) holds up to a multiple, that is,

$$
\bar{h}^{2} \cdot f_{*} c_{2}(X)=k \bar{\theta} .
$$

As before, we pull back this relation to $X$ in order to make computations in cohomology. We get

$$
h^{2} \cdot 2 c_{2}(X)=2 k \bar{\theta} .
$$

Since in cohomology we have $h^{2} \cdot c_{2}(X)=60$, we must have $k=60$, and (5-2) is proved.

In a similar way, we can rewrite (5-3) as

$$
f_{*} c_{2}(X)=15 \bar{h}^{2}-3\left[Y_{A}[2]\right]
$$

and take squares to write $\left(f_{*} c_{2}(X)\right)^{2}$ as a combination of $\bar{h}^{4}$ and a 0 -cycle supported on $Y_{B}$ [2]. This shows that $\left(f_{*} c_{2}(X)\right)^{2}$ is a rational multiple of $\bar{\theta}$.

As usual a cohomology computation yields the precise form

$$
c_{2}(X)^{2}=828 \theta \text {. }
$$

Now we can use Proposition 4.5 to conclude that $c_{4}(X)=k \theta$, and finally we get $k=324$ by comparison with the analogous computation in cohomology. This takes care of all relations in degree 8 . 
The only relation in degree 6 comes from Proposition 2.2, and is

$$
c_{2}(X) \cdot h=5 h^{3} \text {. }
$$

We already proved that the same holds in $C H^{*}(X)$ in Proposition 4.5, so this ends the proof of the main theorem.

\section{References}

[Beauville 2007] A. Beauville, "On the splitting of the Bloch-Beilinson filtration", pp. 38-53 in Algebraic cycles and motives, vol. 2, edited by J. Nagel and C. Peters, London Math. Soc. Lecture Note Ser. 344, Cambridge University Press, 2007. MR 2009c:14007 Zbl 1130.14006

[Beauville and Voisin 2004] A. Beauville and C. Voisin, "On the Chow ring of a $K 3$ surface", $J$. Algebraic Geom. 13:3 (2004), 417-426. MR 2005b:14011 Zbl 1069.14006

[Ferretti 2009] A. Ferretti, The Chow ring of double EPW sextics, thesis, La Sapienza Università di Roma, 2009. To appear in Rend. Mat. Appl. (7), 31:3-4, http://www.mat.uniroma1.it/ricerca/ rendiconti/rendiconti.html.

[Ferretti 2012] A. Ferretti, "Special subvarieties of EPW sextics", Math. Z. (2012). To appear.

[Fulton 1984] W. Fulton, Intersection theory, Ergebnisse der Mathematik (3) 2, Springer, Berlin, 1984. MR 85k:14004 Zbl 0541.14005

[Göttsche 1990] L. Göttsche, "The Betti numbers of the Hilbert scheme of points on a smooth projective surface”, Math. Ann. 286 (1990), 193-207. MR 91h:14007 Zbl 0679.14007

[Griffiths and Harris 1978] P. Griffiths and J. Harris, Principles of algebraic geometry, Wiley, New York, 1978. MR 80b:14001 Zbl 0408.14001

[Hartshorne 1977] R. Hartshorne, Algebraic geometry, Graduate Texts in Mathematics 52, Springer, New York, 1977. MR 57 \#3116 Zbl 0367.14001

[O’Grady 2006] K. O'Grady, “Irreducible symplectic 4-folds and Eisenbud-Popescu-Walter sextics”, Duke Math. J. 134:1 (2006), 99-137. MR 2007e:14062 Zbl 1105.14051

[O'Grady 2008a] K. O'Grady, "Dual double EPW-sextics and their periods", Pure Appl. Math. Q. 4:2 (2008), 427-468. MR 2009b:14076 Zbl 1152.14010

[O’Grady 2008b] K. O'Grady, "Irreducible symplectic 4-folds numerically equivalent to $(K 3)^{[2] \text { ", }}$ Commun. Contemp. Math. 10:4 (2008), 553-608. MR 2009f:32037

[O’Grady 2010] K. O’Grady, “EPW-sextics: taxonomy”, preprint, 2010. arXiv 1007.3882

[Verbitsky 1996] M. Verbitsky, "Cohomology of compact hyper-Kähler manifolds and its applications”, Geom. Funct. Anal. 6:4 (1996), 601-611. MR 98a:53069

[Voisin 2008] C. Voisin, "On the Chow ring of certain algebraic hyper-Kähler manifolds", Pure Appl. Math. Q. 4:3 (2008), 613-649. MR 2009m:14004 Zbl 1165.14012

[Welters 1981] G. E. Welters, Abel-Jacobi isogenies for certain types of Fano threefolds, Mathematical Centre Tracts 141, Mathematisch Centrum, Amsterdam, 1981. MR 84k:14035 Zbl 0474.14028

Communicated by Ravi Vakil

Received 2010-10-20 Revised 2011-01-19 Accepted 2011-02-22

ferrettiandrea@gmail.com Laboratoire Paul Painlevé, Université Lille1, 59655 Villeneuve d'Ascq CEDEX, France http://www.andreaferretti.it/ 


\section{Algebra \& Number Theory}

msp.berkeley.edu/ant

\section{EDITORS}

MANAGING EDITOR

Bjorn Poonen

Massachusetts Institute of Technology

Cambridge, USA

\author{
EDITORIAL BOARD CHAIR \\ David Eisenbud \\ University of California \\ Berkeley, USA
}

\section{BOARD OF EDITORS}

Georgia Benkart

Dave Benson

Richard E. Borcherds

John H. Coates

J-L. Colliot-Thélène

Brian D. Conrad

Hélène Esnault

Hubert Flenner

Edward Frenkel

Andrew Granville

Joseph Gubeladze

Ehud Hrushovski

Craig Huneke

Mikhail Kapranov

Yujiro Kawamata

János Kollár

Yuri Manin

Barry Mazur

Philippe Michel

Susan Montgomery
University of Wisconsin, Madison, USA

University of Aberdeen, Scotland

University of California, Berkeley, USA

University of Cambridge, UK

CNRS, Université Paris-Sud, France

University of Michigan, USA

Universität Duisburg-Essen, Germany

Ruhr-Universität, Germany

University of California, Berkeley, USA

Université de Montréal, Canada

San Francisco State University, USA

Hebrew University, Israel

University of Kansas, USA

Yale University, USA

University of Tokyo, Japan

Princeton University, USA

Northwestern University, USA

Harvard University, USA

École Polytechnique Fédérale de Lausanne

University of Southern California, USA
Shigefumi Mori

Raman Parimala

Jonathan Pila

Victor Reiner

Karl Rubin

Peter Sarnak

Joseph H. Silverman

Michael Singer

Ronald Solomon

Vasudevan Srinivas

J. Toby Stafford

Bernd Sturmfels

Richard Taylor

Ravi Vakil

Michel van den Bergh

Marie-France Vignéras

Kei-Ichi Watanabe

Andrei Zelevinsky

Efim Zelmanov
RIMS, Kyoto University, Japan

Emory University, USA

University of Oxford, UK

University of Minnesota, USA

University of California, Irvine, USA

Princeton University, USA

Brown University, USA

North Carolina State University, USA

Ohio State University, USA

Tata Inst. of Fund. Research, India

University of Michigan, USA

University of California, Berkeley, USA

Harvard University, USA

Stanford University, USA

Hasselt University, Belgium

Université Paris VII, France

Nihon University, Japan

Northeastern University, USA

University of California, San Diego, USA

\section{PRODUCTION}

contact@msp.org

Silvio Levy, Scientific Editor

See inside back cover or www.jant.org for submission instructions.

The subscription price for 2012 is US \$175/year for the electronic version, and \$275/year (+\$40 shipping outside the US) for print and electronic. Subscriptions, requests for back issues from the last three years and changes of subscribers address should be sent to Mathematical Sciences Publishers, Department of Mathematics, University of California, Berkeley, CA 94720-3840, USA.

Algebra \& Number Theory (ISSN 1937-0652) at Mathematical Sciences Publishers, Department of Mathematics, University of California, Berkeley, CA 94720-3840 is published continuously online. Periodical rate postage paid at Berkeley, CA 94704, and additional mailing offices.

ANT peer review and production are managed by EditFLOW ${ }^{\circledR}$ from Mathematical Sciences Publishers.

PUBLISHED BY

mathematical sciences publishers

http://msp.org/

A NON-PROFIT CORPORATION

Typeset in IAT $_{\mathrm{E}} \mathrm{X}$

Copyright (C2012 by Mathematical Sciences Publishers 


\section{Algebra \& Number Theory}

Volume $6 \quad$ No. $3 \quad 2012$

The image of complex conjugation in $l$-adic representations associated to automorphic forms

\section{RICHARD TAYLOR}

Betti numbers of graded modules and the multiplicity conjecture in the

non-Cohen-Macaulay case

\section{MATS BOIJ and JONAS SÖDERBERG}

$\mathscr{L}$-invariants and Shimura curves

SAMIT DAsGupta and MATTHEW GREENBERG

On the weak Lefschetz property for powers of linear forms

Juan C. Migliore, Rosa M. Miró-Roig and Uwe Nagel

Resonance equals reducibility for $A$-hypergeometric systems

Mathias Schulze and Uli WaLther

The Chow ring of double EPW sextics

ANDREA FERRETTI

A finiteness property of graded sequences of ideals

Mattias JONSSON and Mircea MustaȚĂ

On unit root formulas for toric exponential sums

Alan Adolphson and SteVen Sperber

Symmetries of the transfer operator for $\Gamma_{0}(N)$ and a character deformation of the 